\title{
APLICACIÓN DE METODOLOGÍA DE MARCO LÓGICO PARA EL ANÁLISIS DEL PROGRAMA NACIONAL DE PESQUISA Y CONTROL DEL CÁNCER CERVICOUTERINO EN CHILE
}

\author{
Sandra Lanza S., Carolina Sepúlveda V., Macarena Olate B., César Espejo C. \\ Médicos Residentes, Cátedra de Medicina Familiar, Universidad de Valparaíso. Alumnos de Magíster de Salud Pública, \\ Universidad de Valparaíso.
}

\section{RESUMEN}

La metodología de marco lógico (MML) es una herramienta de gestión utilizada para el diseño, monitoreo y evaluación de proyectos y programas. Promovida por el Banco Mundial y usada por la Dirección de Presupuestos de Chile, desde 1997. Algunos de los actuales programas de salud de nuestro país, se remontan a épocas en que esta metodología no estaba en boga. En el presente trabajo, se analiza utilizando esta metodología, el Programa de Pesquisa y Control de Cáncer Cervicouterino, vigente en Chile desde 1987. Este método nos permitió detectar posibles áreas falentes, principalmente en la definición de la población objetivo, en la explicitación del presupuesto, en la coordinación de los diferentes niveles del programa y en la participación comunitaria. Se presentan algunas propuestas que podrían contribuir al mejor desarrollo de este programa.

\section{PALABRAS CLAVE: Metodología, marco lógico, evaluación, programa, cáncer cervicouterino}

\section{SUMMARY}

The methodology of logical framework (LFW) is a management tools used to design and evaluation of projects and programs. Promoted by de World Bank and used by the Direction of Budgets of Chile, since 1997. The projects and programs on health force in our country, mostly, dating back to times when this methodology it was not in vogue. In this work, using this methodology, we analyze the Program of Control of Cervical Cancer in Chile, since 1987. This method allowed us to detect possible lack areas, mainly in the definition of the target population, in the clarification of budget, in the coordination of the different levels of the program and the community participation. Finally, showed some proposals that could contribute to better development of this program.

KEY WORDS: Methodology, logical framework, evaluation, program, cervical cancer

\section{INTRODUCCIÓN}

La metodología de marco lógico (MML) es utilizada por organismos multilaterales de desarrollo como el Banco Mundial y el Banco Interamericano de Desarrollo. Permite la conceptualización, dise- ño, ejecución y evaluación de planes y programas. Identifica los objetivos de los programas, para luego determinar la consistencia de su diseño y resultados con esos objetivos, y permite concatenar las diferentes etapas siguiendo una lógica horizontal y vertical. 
En Chile desde 1997, la Dirección de Presupuesto (DIPRES) comenzó la evaluación de programas públicos, a través de la línea denominada "Evaluación de Programas Gubernamentales" utilizando la MML.

Se analizó utilizando la MML el "Programa de Pesquisa y Control de Cáncer Cervicouterino" vigente en nuestro país. Esta investigación fue realizada con fines académicos durante el desarrollo del Magíster de Salud Publica del cual somos alumnos, por lo tanto declaramos no presentar conflictos de interés.

El "Programa Nacional de Pesquisa y Control del Cáncer Cervicouterino", se enmarca dentro de las políticas de salud del gobierno de Chile, cuyo diseño, planificación y regulación es responsabilidad del Ministerio de Salud (Minsal). Ellas responden al mandato de la Constitución Política del Estado que establece en su artículo 19, № 1, "el derecho a la vida y a la integridad física y psíquica de las personas" (1).

En los países en desarrollo, el cáncer cervicouterino $(\mathrm{CaCu})$ constituye un importante problema de salud pública. En Chile, los esfuerzos para una detección precoz del $\mathrm{CaCu}$ se iniciaron en 1966, a través de un convenio con la Facultad de Medicina de la Universidad de Chile con apoyo de la OPS/OMS, realizando el Papanicolaou (PAP) en Atención Primaria de Salud (APS) anualmente a las mujeres usuarias del programa materno-infantil.

En 1987, luego de la evaluación de las medidas implementadas, fueron reformuladas y se constituyó el Programa Nacional de Pesquisa y Control del Cáncer Cervicouterino. Dentro de las prestaciones del programa, el PAP debe realizarse cada 3 años y la población objetivo está focalizada en mujeres entre 25 y 64 años, con énfasis en las mayores de 35 años, asegurando la oportunidad y confiabilidad del examen citológico y el tratamiento de los casos detectados.

Entre 1988 y 1994, estas estrategias se aplicaron en forma piloto en la Región Metropolitana y luego de evaluarse fue implementada en el resto del país. En 1997, el CaCu se definió como prioridad programática país. Desde entonces la cobertura de PAP vigente en mujeres beneficiarias entre 25 a 64 años, ha aumentado de $26 \%$ a $68 \%$ entre 1990 y 2004 respectivamente (2). Se ha demostrado un descenso sostenido de la mortalidad por éste cáncer, especialmente en los grupos de edad definidos como foco del programa, mostrando un descenso de un $48,3 \%$ en el grupo de 25 a 64 años entre los años 1987 y 2003 y un descenso de 52,2\% en el grupo de más alto riesgo, esto es de 35 a 64 años en el mismo periodo (3).

El 1 de julio de 2005, se inició la Reforma de Sa- lud, mediante el Régimen General de Garantías en Salud contenidas en la ley № 19.966, garantizando la oportunidad del diagnóstico, tratamiento, seguimiento y protección financiera de 25 patologías, entre ellas, el $\mathrm{CaCu}$. Dentro de los objetivos sanitarios del Minsal para el periodo 2000-2010 con respecto al $\mathrm{CaCu}$, se estableció reducir en $40 \%$ la tasa de mortalidad al término de la década (4).

Esta patología es responsable de la mayor pérdida de años de vida saludable en la mujer, teniendo el 2006 el primer lugar en la tasa de años de vida potencialmente perdidos, 129 por 100.000 mujeres, con gran impacto social y económico, ya que afecta a mujeres relativamente jóvenes, en edad productiva y reproductiva (5). Ese mismo año ocupó el quinto lugar entre las muertes por cáncer en la mujer, siendo la tasa de mortalidad de 8,1 por 100.000 mujeres (6). La mortalidad en mujeres menores de 25 años es ocasional y el ascenso se produce a partir de los 35 años, aumentando progresivamente. El $52,6 \%$ de las muertes se produce en mujeres entre 35 y 64 años (3).

En APS, cada centro de salud tiene un encargado de programa, que vela por el cumplimiento de estrategias en la comunidad y logro de metas establecidas. En el nivel secundario existe el laboratorio de referencia nacional de citopatología, responsable del control de calidad de los otros 22 laboratorios ubicados en el país. En el nivel terciario, en cada hospital funciona una Unidad de Patología Cervical (UPC) con un equipo capacitado. Se cuenta con una red de derivación para quimioterapia y radioterapia. En cada Servicio de Salud se desempeña un médico y una matrona encargados de la gestión del programa.

Cuando el resultado del examen es informado como PAP (+) o existe sospecha clínica de un $\mathrm{CaCu}$, se debe derivar al Nivel Secundario de Atención, a la UPC, para confirmación diagnóstica, tratamiento y seguimiento del caso. Cada uno de estos pasos tiene garantizados prestaciones y plazos definidos.

La incidencia estimada por la Unidad de Cáncer del Minsal, se basa en la evaluación informatizada anual de los 28 Servicios de Salud del Sistema Público, pero no se tiene información de los casos nuevos generados por el Sistema Privado de Salud (7).

Es fundamental identificar la población de riesgo para optimizar los recursos y conseguir verdadero impacto. El objetivo es la realización del $95 \%$ de los PAP a la población objetivo y un $5 \%$ de ellos a otros grupos etarios con factores de riesgo identificados. La cobertura de PAP vigente en la población beneficiaria se ha incrementado progresivamente, pero se sabe que para lograr un verdadero impacto 
en la mortalidad se deben lograr en forma sostenida coberturas iguales o mayores a $80 \%$ (8).

\section{IDENTIFICACIÓN DE LA POBLACIÓN BENEFI- CIARIA}

Se cuantificó la población femenina chilena (Tabla I) y luego la población femenina beneficiaria de FONASA, el programa explicita su orientación a este grupo de mujeres (Tabla II). Para este programa, la población objetivo de cada uno de los componentes, explicadas a continuación, son distintas.
Tabla I

POBLACIÓN GENERAL Y POR SEXO, SEGÚN CENSO 2002 - CHILE

\begin{tabular}{lr}
\hline Población total Chile & 15.116 .435 \\
Población total Mujeres & 7.668 .740 \\
Población total Hombres & 7.447 .695
\end{tabular}

Fuente: INE. Hallado en www.ine.cl (acceso en enero 2010).

Tabla II

POBLACIÓN DE REFERENCIA, POTENCIAL Y OBJETIVO EN BASE A POBLACIÓN BENEFICIARIA DEL FONASA ESTIMADA A DICIEMBRE DE 2008

Población de referencia : total de beneficiarios FONASA

12.248.257

Población potencial: mujeres

6.225 .695

Población sin problemas: hombres

6.022 .562

Población objetivo Componente 1: mujeres entre 25 y 64 años

3.042 .658

Población potencial postergada:

- mujeres $<25$ años $y>65$ años

3.183.037

I mujeres $<25$ años

2.423.977

- mujeres $>65$ años

759.060

Población objetivo Componente 2:

Total

Datos no

Etapa precoz

encontrados

Etapa avanzada

Población objetivo Componente 3:

Total

Datos no

Etapa precoz

encontrados

Etapa avanzada

Población de referencia : Total de beneficiarios FONASA

Población potencial: mujeres

Población sin problemas: hombres

6.022 .562

Población objetivo: mujeres entre 25 y 64 años

3.042 .658

Población potencial postergada:

- mujeres $<25$ años $y>65$ años

3.183.037

- mujeres <25 años

- mujeres >65 años

759.060

Fuente: FONASA. Hallado en www.fonasa.cl (acceso en enero 2010). 
Componente 1: La población objetivo de este componente está constituida por todas las mujeres entre 25 y 64 años beneficiarias del sistema público de salud, sobre la cual se construye el indicador de cobertura del PAP.

Componente 2: La población objetivo de este componente está constituida por todas las mujeres entre 25 y 64 años beneficiarias del sistema público de salud, las cuales acceden a atención para confirmación diagnóstica en la UPC. Respecto a esta población, no tuvimos acceso en las fuentes consultadas. Podría haber sido utilizado para su cálculo la incidencia de $\mathrm{CaCu}$, pero este valor considera solamente a las mujeres con biopsia positiva y deja fuera a quienes fueron derivadas por sospecha clínica o PAP positivo y que no fueron confirmadas a través de biopsia.

Componente 3: La población objetivo está constituida por todas las mujeres entre 25 y 64 años beneficiarias del sistema público de salud, las cuales acceden a atención en el nivel terciario de salud con confirmación diagnóstica de lesiones preinvasoras e invasoras. Una opción para objetivar esta población es utilizar las tasa nacional de incidencia de $\mathrm{CaCu}$, sin embargo esto no permitiría realizar una estimación fiable del presupuesto ya que en este componente no solo se incluye a las mujeres diagnosticadas durante el año, si no que hay pacientes que ya estaban siendo tratadas con diagnostico en años anteriores.

\section{PRESUPUESTO}

El financiamiento del programa viene del MINSAL a través de las siguientes vías; per cápita, Programa de prestaciones valoradas (PPV), Programa de prestaciones institucionales (PPI).

Consideramos una dificultad encontrar el presupuesto del programa. Se solicitó información vía correo electrónico a expertos, no obteniendo respuesta. Finalmente, en forma clara y explícita el programa no posee un presupuesto determinado.

A continuación se presenta la información obtenida por componentes, según valor de las prestaciones y la población objetivo:

Componente 1: La única actividad valorizada es la toma del PAP, realizada a la población beneficiaria cada tres años. El valor del PAP se obtiene de FONASA. El resto de las actividades no se encuentran valorizadas ni cuantificadas.

Componente 2: No existen datos fiables respecto al número de prestaciones realizadas. Por lo tanto a pesar que algunas de las actividades de este componente se encuentran valorizadas, no es posible calcular el presupuesto en este ítem.

Componente 3: Se encuentran valorizadas algunas de las actividades, pero como fue expuesto anteriormente la población no puede ser determinada, por lo cual el presupuesto no puede ser calculado.

\section{JUICIO EVALUATIVO}

Actualmente este programa no está elaborado con la MML. Esta evaluación fue realizada, principalmente, a partir de dos insumos; la Guía Clínica de Cáncer Cervicouterino del MINSAL 2005 y el Formulario de evaluación del Programa $\mathrm{CaCu}$. Tanto el fin, como el propósito y componentes del programa, se encuentran descritos en la guía clínica, lo mismo para las actividades de los componentes 2 (Confirmación diagnóstica) y 3 (Tratamiento). Las actividades del componente 1 (Pesquisa y derivación) y todos los indicadores de los tres componentes se extraen del formulario. Los supuestos planteados en la matriz son los que dedujimos del análisis del programa en su conjunto ya que no se encuentran explícitos en ningún documento de los que tuvimos acceso.

La evaluación del Programa se basó en los siguientes aspectos:

a) Justificación de su implementación de acuerdo a impacto sanitario y socioeconómico. Consideramos que el programa $\mathrm{CaCU}$ en Chile tiene plena justificación, por ser una patología prevalente que causa mortalidad importante en la población femenina. Además, cumple con los requisitos para tamizar una enfermedad; historia natural conocida, método de detección simple y de bajo costo, tratamiento de lesiones preinvasoras con $100 \%$ de curación y tratamiento caro, complejo y con baja sobrevida a 5 años.

Con las medidas ministeriales adoptadas desde 1987, se ha logrado subir la cobertura, observándose mejoría en los resultados; mayor incidencia de cáncer in situ por sobre el cáncer invasor y declinación de la curva de mortalidad. A pesar de los avances alcanzados los porcentajes de cobertura en la población beneficiaria no son suficientes para un programa exitoso de pesquisa.

La disminución de la mortalidad por CaCu podría considerarse multicausal, existen estudios en Chile que lo sustentan (9). Una de estas causas seria la implementación de mejores tratamientos a nivel mundial que han sido adoptados por nuestro país, además parecen ser más breves los tiempos de latencia entre las distintas fases del proceso; pesquisa, diagnostico y tratamiento. Es importante el diagnóstico de esta enfermedad en etapas precoces, en el año 2000 el $62 \%$ de los casos nuevos se diagnosticaba en etapa I, en 1987 solo alcanzaba a un 10,85\% (9).

b) Concordancia con la evidencia científica disponible y con la costo-efectividad de las medidas implementadas. La mayoría de los casos de $\mathrm{CaCu}$ 
comienzan con una lesión intraepitelial que puede durar 10 a 20 años hasta la aparición de un cáncer invasor. Estudios de pacientes que han sido seguidas con citología y colposcopia demuestran que la mayoría de las lesiones intraepiteliales de bajo grado regresan espontáneamente, las de alto grado tienen mayor probabilidad de progresar hacia un cáncer invasor.

El tamizaje es una técnica utilizada para la detección de una enfermedad en etapas iniciales, en individuos asintomáticos. Desde 1941, el PAP ha sido utilizado como herramienta de pesquisa en el $\mathrm{CaCu}$. Las mujeres en edad fértil, sin PAP, tienen un riesgo 3 veces mayor de presentar la enfermedad que las mujeres con examen al día.

No existe consenso de cuando empezar el tamizaje, se recomienda con el inicio de la vida sexual o a los 18 años, esto depende del análisis de costo efectividad que haga cada país, en Chile según las cifras de morbilidad se decidió el inicio a los 25 años.

La relativa baja sensibilidad de un PAP aislado aumenta con la repetición del examen en un tiempo adecuado. La sensibilidad aumenta escasamente al realizar el examen anual en relación a cada 2 o 3 años, se debe decidir la mejor opción como política para cada país. El número de PAP normales que se debiera esperar antes de espaciar el examen cada 3 años no está claro.

El programa CaCU nos parece concordante con la literatura y evidencia disponible, hasta ahora el PAP sería el mejor método de pesquisa con que se cuenta, esta pendiente la actualización de la guía GES de CaCU elaborada el 2005 y que debió ser reactualizada en el plazo de dos años, considerando además la difusión de recientes guías clínicas internacionales para el manejo de esta patología ( ).

La relación costo efectividad del programa esta dada principalmente por la realización de un tamizaje que ha permitido la detección de la enfermedad en etapas precoces. No se evidencian estrategias sistemáticas dirigidas a la promoción. Es necesario recordar que una mejora sustancial de este programa pasa por ampliar la cobertura de pesquisa, sobre el $80 \%$ de la población objetivo, condición difícil de alcanzar dadas las dificultades antes enunciadas (11). En la actualidad las metas de cobertura son negociadas en forma anual y localmente utilizando cifras cercanas al $60 \%$.

c) Organización y gestión del programa. Actualmente no existe un único programa que abarque la pesquisa, diagnóstico y tratamiento del $\mathrm{CaCU}$, sino que son dos programas; la pesquisa a cargo exclusivamente de la APS y el diagnóstico y tratamiento a cargo del nivel secundario y terciario respectivamente, esta separación se ha intensificado desde el inicio del programa GES que garantiza desde la confirmación diagnostica (Componente 2) en adelante. En base a la información recolectada, podemos inferir que al inicio el programa se planteó como único, lo que estableció lazos entre los niveles de atención que hasta la fecha se mantienen, pero se ven debilitados. En cuanto al componente 2 , no existe una adecuada contrareferencia que permita el seguimiento de las pacientes.

La idoneidad de los elementos constituyentes del nivel secundario es un hecho en el sistema público institucional. En el sistema privado como en el público de libre elección, no existe un referente único o principal donde se deriven las muestras para ser analizadas (tanto PAP como biopsias) las cuales pueden analizarse sin los estándares adecuados.

La existencia de recurso especialista entrenado presenta dificultades, las que pueden ser geográficas (a favor de las regiones más pobladas: Metropolitana, de Valparaíso y del Biobío), como económicas (éxodo del área pública a privada).

d) Evaluación. Destaca la ausencia de indicadores para los cuatro ámbitos (calidad, eficacia, eficiencia y economía). Existen indicadores que burocratizan el sistema, y no parecen capaces de generar un cambio sustancial en base a ellos, conllevando una pérdida de recursos en su registro y estimación. Cabe destacar la falta de indicadores de promoción y satisfacción usuaria, su análisis permitiría establecer estrategias que probablemente mejorarían las coberturas. La fiabilidad de los datos utilizados y la calidad de los registros es un aspecto importante a considerar.

En cuanto a los medios de verificación, el Sistema de Información de Gestión de Garantías Explicitas de Salud (SIGGES) y el programa Cito-Expert, tienen valor indiscutible a nivel nacional, como también el Formulario de Evaluación del Programa Cáncer Cervicouterino, base de datos oficial del programa, utilizada por todos los servicios de salud del país y en la que se basan las evaluaciones anuales del programa.

El programa carece de supuestos que permitan trabajar adecuadamente en la lógica horizontal de la MML. Los supuestos permiten la justificación ante las dificultades de concretar los objetivos propuestos al momento de la evaluación del programa, y dan un indicio de que el mismo fue diseñado anticipándose a situaciones que son inhabituales, pero posibles de suceder.

e) Recursos financieros. Existe dificultad de acceso a los datos del financiamiento del programa. La obtención de ellos se logra mediante la estimación de la población y esto ocurre solo en el componente 1 . Es llamativo que en un programa con garantías explicitas de salud, esta información no se encuentre 
fácilmente disponible. En relación al financiamiento, los recursos llegan por diferentes vías, esto dificulta la optimización de los recursos y la integración del programa.

\section{PROPUESTAS DE MEJORA}

Las hemos dividido de acuerdo a la utilización de recursos financieros que podrían involucrar su ejecución, nos parece que en la mayoría de los casos es el factor limitante para el desarrollo de nuevas estrategias.

Recomendaciones que no involucran recursos adicionales importantes:

- Priorización y desecho de indicadores en pro de garantizar el registro de los de mayor impacto.

- Diseño y utilización de indicadores que permitan evaluar efectivamente este programa desde todos los ámbitos (eficacia, eficiencia, calidad, economía).

- Optimización de los registros, con capacitaciones periódicas y auditorias.

- Detección de brechas de recursos humanos y económicos.

- Optimización del funcionamiento de la red, mejorando la contrarreferencia hacia APS.

- Estrategias sistemáticas de promoción con diseño participativo, de usuarios internos y externos.

- Incentivos no pecuniarios (tarde libre, reconocimiento ante pares) a los equipos por cumplimiento de metas de cobertura.

- Reformulación del programa con la MML que incluya actualización de la guía GES.

Recomendaciones que involucran recursos adicionales importantes:

- Optimización de los sistemas informáticos que permitan una efectiva integración de la red tanto del sistema público y privado.

- Asignación de recursos con presupuesto único usando la MML.

- Capacitación de los equipos según las brechas detectadas.

- Política de incentivos económicos a los médicos del sistema privado y público libre elección, para mejorar la cobertura, notificación y registro de los datos.

\section{CONCLUSIONES}

Consideramos que el Programa de Pesquisa y Control de Cáncer Cervicouterino, ha respondido de manera adecuada frente al problema que esta patología origina a nuestra población, encontrándose plenamente justificado. Sin embargo, requiere de algunas modificaciones en pos de la actualización de la evidencia científica utilizada, la moderni- zación de la metodología tanto en lo que se refiere a detección participativa de necesidades, como a los sistemas de registro y canales de comunicación.

Creemos que la unificación del programa, tanto en el sentido de su división entre los distintos niveles de atención, entre lo GES y no GES, entre lo público y privado y sus distintas fuentes de financiamiento, llevarían a la potenciación de sus virtudes lo que se reflejaría probablemente en un mayor impacto en la salud de la población.

\section{BIBLIOGRAFÍA}

1. Gobierno de Chile. Minsal. Informe Final de Evaluación Programa Salud Mental. Ministerio de Hacienda. Dirección de Presupuestos, Junio 2002.

2. Prieto M. Presentación del Programa nacional de pesquisa y control del cáncer cervicouterino del Ministerio de Salud del Gobierno de Chile. México. 2008. Hallado en: URL: http://www.sabin.org/files/marta_prieto.pdf

3. Gobierno de Chile. Ministerio de Salud. Guía Clínica: "Examen de medicina preventiva", 2008. Hallado en: www.redsalud.gov.cl/archivos/guiasges/Guia_ EMP_100108.pdf

4. Gobierno de Chile. Ministerio de Salud. Los objetivos sanitarios para la década 2000-2010. Hallado en: http://epi.minsal.cl/epi/html/elvigia/vigia15.pdf

5. Sepúlveda P, González F, Napolitano C, Roncone E, Cavada G. Cáncer de cuello uterino: sobrevida a 3 y 5 años en Hospital San José. Rev Chil Obstet Ginecol 2008;73(3):151-4.

6. Departamento de Estadísticas e Información de Salud, Ministerio de Salud. 2008. Hallado en: URL: www.deisminsal.cl

7. Gobierno de Chile. Ministerio de salud. Objetivos Sanitarios para la Década 2000-2010: Estado de avance en los objetivos de impacto y desarrollo. Capítulo I: Mejorar los logros sanitarios alcanzados. 2005. p.41. Hallado en: URL: http://epi.minsal.cl/epi/html/sdesalud/OS/EVOS_cap_II.pdf

8. Pérez $C$. Análisis espacial y temporal del riesgo de muerte por cáncer cervicouterino en Chile: 19902004. Trabajo de titulación para obtener el título de Ingeniero en Ejecución de Ambiente. Universidad de Santiago de Chile. 2006.

9. Capurro I, Rojo J, Pino T, Vásquez C, Garay J, Venegas M. Programa de detección y control de cáncer de cuello uterino en Servicio de Salud Araucanía Sur. Rev Chil Obstet Ginecol 2002;67(2):114-20.

10. Guía Clínica: American Society for Colposcopy and Cervical Pathology, actualización 2009.

11. Quinn M, Babb P, Jones J, Allen E. Effect of screening on incidence and mortality from cervical cancer in England: evaluation based on routinely collected statistic. BMJ 1999;318(7188):904-8. 